\title{
Thai elephant-assisted therapy program
}

\begin{abstract}
The objective of this article is to introduce a new treatment program for children with autism, the Thai Elephant-assisted Therapy Program (TETP). Research and results were provided as well as the reasons of using elephant as a therapeutic medium. Some activities in the TETP are presenting via pictures.
\end{abstract}

Keywords: children with autism, children with special needs, poor socialization, bruininks oseretsky test, adaptive behavior
Volume 2 Issue 2 - 2017

\author{
Nuntanee Satiansukpong, Maethisa (Arisa) \\ Pongsaksri and Daranee Sasat \\ Department of Occupational Therapy, Chiang Mai University, \\ Thailand
}

Correspondence: Nuntanee Satiansukpong, Department of Occupational Therapy, Chiang Mai University, Thailand, Tel 6688 207 9727, Email nuntanee.s@cmu.ac.th

Received: March 23, 2017| Published: April 24, 2017
Abbreviations: TETP, thai elephant-assisted therapy program; PDD, pervasive developmental disorder; AAT, animal assisted therapy; ASD, autistic spectrum disorder

\section{Introduction}

The objective of this article is to introduce a new treatment program for children with autism, the Thai Elephant-assisted Therapy Program (TETP). Research and results were provided as well as the reasons of using elephant as a therapeutic medium. Some activities in the TETP are presenting via pictures. Autism is a severe developmental disorder, classified by DSM V, the subtype in Pervasive Developmental Disorder (PDD). Its significant symptoms are poor socialization, communication difficulty and restricted interest problems. ${ }^{1}$ Over six decades, many studies have been performed to investigate the reason for autism, but no study has identified its root cause. Only the potential causes such as genetic and environment ones have been identified. In the past decade, the number of individuals with autism has increased dramatically. In the USA during 2007, the Autism Society of America reported a rising prevalence rate of up to $1: 150$, with Thailand having more than 200,000 individuals with autism.

Many treatment methods have been developed and used for intervention in order to alleviate problems found in persons with autism. However, results of the treatment have been controversial because no two individuals with autism present the same difficulties. Animalassisted therapy (AAT) also known as pet therapy is the introduction of an animal into the immediate surroundings of an individual. ${ }^{2}$ The animal is used as a medium of interaction with a therapeutic purpose. Previous studies of AAT revealed many physiological and psychological benefits for example calming influence, decreased heart rate and blood pressure, reduced fear and anxiety, increased strength and improved cognition and balance..$^{3-5}$ Occupational Therapy (OT) has a history of using animal as a part of therapeutic intervention, but few studies have investigated AAT from an OT perspective. This is an attempt to use Thai elephants in the treatment program for autistic individuals for three reasons.

Firstly, the Thai elephant is relevant to Thai culture. In the past, Thai elephants had a significant role in war and became a valuable symbol for the people of Thailand. Secondly, activities with Thai elephants fit with the Model of Practice in OT. Thai elephants are used as therapeutic media and also companions in doing activities with each autistic individual. The large size of the elephant may attract the interest of the subjects and motivate them to interact with elephants and the environment. ${ }^{6,7}$ The elephant also eats a large amount of food give the individual with autism more opportunity to interact with and care for them. An elephant can perform other interactions, for example, if someone drops article such as a shoe or wallet while riding it animal would pick it up and return it to the person. Riding and bathing elephants are also relevant activities in the model of sensory integration because they provide tactile, proprioceptive and vestibular inputs. ${ }^{8}$ Thirdly, the information obtained from experts in the Thai Elephant Conservation Center is that Thai elephants are clever and caring animals. They also are easily trained to assist in the treatment program.

At Thai Elephant Conservation Center, the researchers examined the feasibility of using Thai elephants in the treatment program. Feasibility was defined by whether a treatment program could be implemented. The purpose of this study was to investigate the effects of a new treatment program incorporating Thai elephants as a means of providing new occupations as well as meaningful activities with sensory information for an individual with autism. The session of new TETP was from 9.00AM-4.00PM. It provided a whole day session of intensive treatment, 4 days a week, for a total of 12 days in 3 weeks. There were 8 activities: orientation, fetching and taking elephant, buying elephant food and feeding the elephant, bathing the elephant, riding the elephant, playing games with the elephant (e.g. throwing ball, running race, bowling), doing elephant arts and craft and relaxation. These activities were implemented by occupational therapists. The research questions were as follows: Was the Thai elephant-assisted Therapy Program feasible for an individual with autism? Would the individual with autism demonstrate improved sensory processing and adaptive behavior after 3 weeks of the Thai elephant-assisted Therapy Program? The Short Sensory Profile ${ }^{9}$ the Balance Subtest of the Bruininks Oseretsky Test, ${ }^{10}$ the interview edition survey form of the Vinland Adaptive Behavior Scales ${ }^{11}$ were measured a week before and after the TETP. 


\section{Past research projects}

The first project, entitled "Thai Elephant-assisted Therapy Program (TETP) the Feasibility in Assisting an Individual with Autism," was a pilot study to examine the feasibility of the TETP in assisting individuals with autism. ${ }^{12}$ The TETP was an intensive program comprising 4 days a week within the duration of 3 weeks. The change of pretest and post-test showed that sensory processing, balance and adaptive behavior composite were improved in all cases $(n=4)$. This project was orally presented at the Occupational Therapy Symposium in June 2008 in the NTU Building, Singapore. The paper won "The Best Paper Presentation Prize" from the Singapore and Malaysian Occupation Therapy Association. This paper was published in the World Federation of Occupational Therapy Bulletin in November 2008. This project also achieved the first runner up prize in the category of Best Innovation for Individuals with Disability from the Chiang Mai Public Health Organization in August 2009. These past achievements would not have been possible if the benefits of the TETP were not addressed. The program was used to promote social and adaptive behavior among individuals with autism. Consequently, their lives will become more joyful and less dependent.

The second project was initially conducted with a larger number of participants. There were 8 individuals with autism recruited some of the children with autism go to school during weekdays therefore the TETP was designed to provide only weekend sessions. The participants were attended the TETP every weekend for the duration of 6 weeks. The purpose of the program was to investigate adaptive behavior and its generalization for use in a school setting. The behavior of autistic individuals at school was recorded before, during and after receiving the TETP. The mean adaptive behavior composite from Vineland Adaptive Behavior Scales between pre test (309.25 \pm 42.67$)$ and post test (323.57 \pm 34.00$)$ was significantly different at $\alpha=0.05$ $(\mathrm{p}=0.17)$ Table 1 . In the third project, children with autistic spectrum disorder $[\mathrm{ASD}]$ were recruited and received one of the two treatment programs. Researchers created two new Thai Elephant-Assisted Therapy Programs, which were more specific to the problems found in individuals with autistic spectrum disorder [ASD]. One program promoted social skills and reduced maladaptive behavior, while the other promoted motor planning. The new TETP were provided for 2 groups of individuals having ASD. Both groups received the new TETP in different protocols: 3 weeks or 6 weeks: The results of the 3 week duration program were compared with those of the 6 week one. The results showed that effective size of the short and long programs in maladaptive behavior were 0.9 and 0.6 respectively. In social domain, the effective size of the short and long programs was equal at 0.5 . This research was financed by an office of the National Research Council of Thailand. It was collaboration between the Department of Occupational Therapy, Faculty of Associated Medical Sciences, Chiang Mai University and the Thai Elephant Conservation Center, Forest Industry Organization. The result showed that there was not a significant difference between the 3 weeks or 6 weeks of treatment $\mathrm{p}>0.05$.

The fourth project, entitled "Thai elephant Assisted Therapy Program in Children with Down syndrome," was published in the Journal of Occupational Therapy International. ${ }^{13}$ Sixteen children with Down syndrome were recruited and were separated into 2 groups: control and experiment. The control group got regular school activities, while the experimental group got additional Thai Elephant Assisted Therapy Program for Down syndrome twice a week for 8 week duration. The Berry VMI ${ }^{14}$ the postural control record form were tested before and after the treatment program. The results showed that the visual motor integration of the experimental group was significantly different from that of the control group $(\mathrm{U}=13.50$, $\mathrm{p}=0.04$ ). Activities in Thai Elephant Assisted Therapy Program are composed of activities such as buying elephant food, feeding elephants, bathing elephants, playing games with elephants, riding elephants, elephant arts and crafts and relaxation. Some of these activities are shown in Figure 1-9.

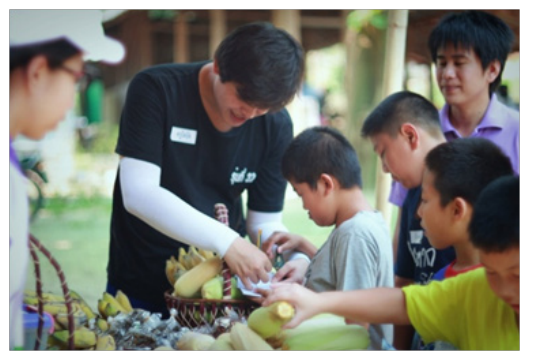

Figure I Buying sugar cane, banana, water melon, cereal, sunflower seed etc. for an elephant buddy.

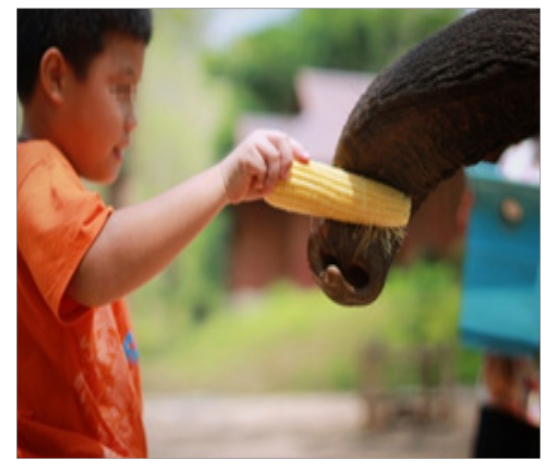

Figure 2 A Child focuses on an elephant while feeding it with corn.

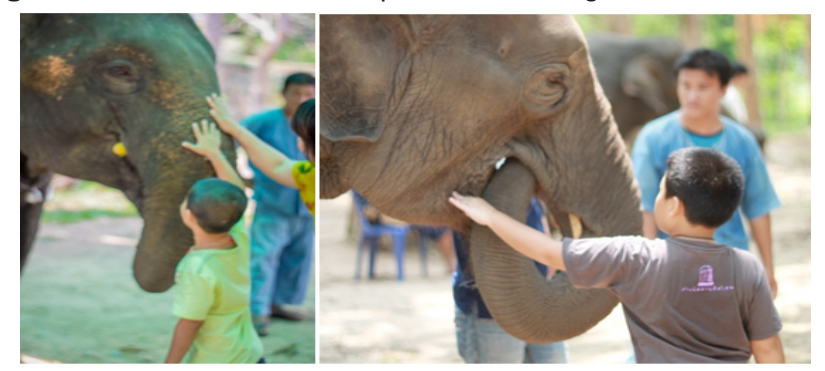

Figures 3\& 4 Touching an elephant is a new experience.

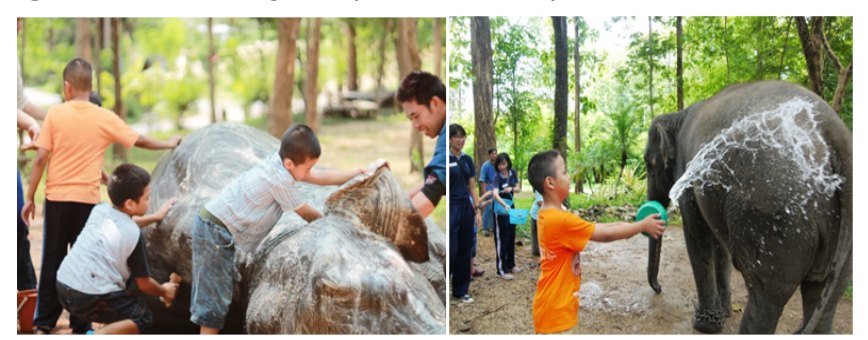

Figures 5 \& 6 Taking care and bathing an elephant as a new role for children. 


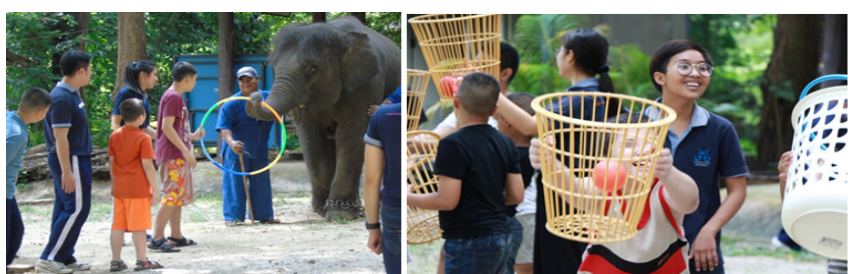

Figures 7 \& 8 Playing a game with an elephant is a means to learn: stay with friends and express feelings.

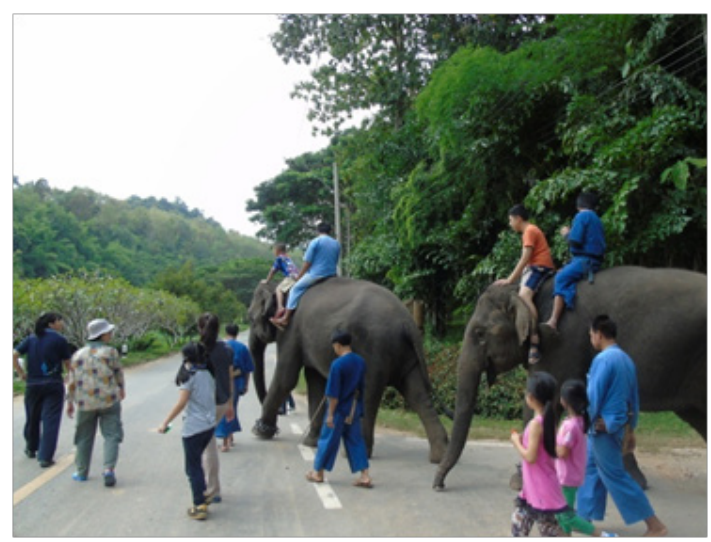

Figure 9 Riding an elephant is a means to learn motor planning as well as improving motor control and balance.

\section{Results}

The elephants were used as therapeutic media and companions in doing activities with children with autism. The first project study showed that participants' post-test scores were higher than the pretest scores in the Short Sensory Profile, balance subtest and adaptive behavior composite as shown in Tables 1-3. They were focused on activities at hand and improved in interaction with therapists and peers. Most of them love feeding, bathing, riding the elephant Tables $2-4$.

Table I Adaptive behavior mean and standard deviation from the Vineland Adaptive Behavior Scales measured before and after the TETP. $(n=7)$ by using Wilcoxon signed ranks test

\begin{tabular}{lllll}
\hline \multirow{2}{*}{$\begin{array}{l}\text { Adaptive } \\
\text { behavior }\end{array}$} & Vineland adaptive behavior scale & $\mathbf{n}$ & p value \\
\cline { 2 - 5 } & Pretest $(\overline{\mathbf{x}} \pm$ S.D. $)$ & $\begin{array}{l}\text { Posttest } \\
(\overline{\mathbf{x}} \pm \text { S.D. })\end{array}$ & & \\
\hline $\begin{array}{l}\text { Communication } \\
\text { Daily living }\end{array}$ & $97.75 \pm 19.88$ & $98.43 \pm 15.71$ & 7 & 0.553 \\
$\begin{array}{l}\text { Activity } \\
\text { Social Skill }\end{array}$ & $129.75 \pm 18.86$ & $134.14 \pm 18.31$ & 7 & $.046 * *$ \\
Total & $81.75 \pm 14.70$ & $91.00 \pm 13.44$ & 7 & $.041 * *$ \\
\hline
\end{tabular}

Table 2 The score of each individual with autism from the Short Sensory Profile before and after the TETP as well as \% Change of the score between pretest and posttest

\begin{tabular}{llll}
\hline Case No. & Pretest & Posttest & \%Change \\
\hline 1 & 144 & 177 & 18.6 \\
2 & 137 & 153 & 10.4 \\
3 & 146 & 152 & 3.9 \\
4 & 141 & 151 & 6.6 \\
\hline
\end{tabular}

Table 3 The balance of each individual with autism in terms of total point score at pretest-posttest and age equivalent from the Bruininks Oseretsky Test as well as \% Change of the score between pretest and posttest

\begin{tabular}{llll}
$\begin{array}{l}\text { Case no. } \\
\text { (Age*) }\end{array}$ & Total point & $\begin{array}{l}\text { Age equivalent } \\
\text { (years-months) }\end{array}$ \\
\hline $1(17-11)$ & Pretest & 15 & 11-Apr \\
& Posttest & 22 & 11-Jun \\
& $\%$ Change & 31.8 & \\
$2(11-1)$ & Pretest & 8 & 2-Apr \\
& Posttest & 17 & 2-May \\
& $\%$ Change & 52.9 & \\
$3(12-7)$ & Pretest & 13 & 5-Apr \\
& Posttest & 20 & 2-Jun \\
& $\%$ Change & 35 & \\
$4(14-10)$ & Pretest & 11 & 2-Apr \\
& Posttest & 18 & 5-May \\
& $\%$ Change & 38.8 & \\
\hline
\end{tabular}

Table 4 The standard score (SS), adaptive levels (AL), and age-equivalent (AE) in year-month of the adaptive behavior composite from the Vineland Adaptive Behavior Scales measured before and after the TETP

\begin{tabular}{lllllll}
\hline Case No. $\left(\right.$ Age $\left.^{*}\right)$ & \multicolumn{9}{l}{ Adaptive behavior } \\
\hline & Pretest & \multicolumn{5}{l}{ Posttest } \\
\hline $1(17-11)$ & 34 & Lo & 5-Jun & 38 & Lo & 7-Jul \\
$2(11-1)$ & 56 & Lo & 8-Jun & 59 & Lo & 10-Jun \\
$3(12-7)$ & 51 & Lo & 3-Jun & 54 & Lo & 10-Jun \\
$4(14-10)$ & 40 & Lo & 1-Jun & 40 & Lo & 2-Jun \\
\hline
\end{tabular}

\section{Limitation and recommendation}

Previous research projects studied only the short term effects of TETP (3, 6, 8 weeks). For future studies, the long term effects of the TETP applied to extended periods of time (i.e. 3 and 6 months after TETP, etc.) to confirm TETP effectiveness. In addition, the research studies were conducted with Thai children with autism who were familiar with elephants. It was not conducted in other country samples. It might be of interest to see whether the TETP works well with children who are not familiar with elephants. Even though the cost of the TETP is a lot more expensive when compared to a traditional OT treatment program, significant behavior improvement in children were found in the short term. Future research studies should explore the TETP with different ethnic background participants. Funding for running the TETP is needed to help support parents of children with autism.

\section{Acknowledgements}

None.

\section{Conflict of interest}

The author declares no conflict of interest. 


\section{References}

1. American Psychiatric Association. Diagnostic and statistical manual of mental disorders: DSM-5. Washington, USA: American Psychiatric Publishing; 2013.

2. Velde BP, Cipriani J, Fisher G. Resident and therapist views of animalassisted therapy: Implications for occupational therapy practice. Australian occupational Therapy Journal. 2005;52(1):43-50.

3. Barker SB, Pandurangi AK, Best AM. Effects of animal-assisted therapy on patients' anxiety, fear and depression before ECT. J ECT. 2003;19(1):3844.

4. Baun MM, Oetting K, Bergstom N. Health Benefits of companion animals in relation to the physiologic indices of relaxation. Holist Nurs Pract. 1991;5(2):16-23.

5. Connor K, Miller J. Help from our animal friends. Nurs Manage. 2000;31(7):42-46.

6. Kielhofner G. A model of human occupation: Theory and application. Baltimore, USA: Lippincott Williams \& Wilkins; 1985.

7. Bracher M. Therapeutic horse riding: What has this to do with occupational therapists? British Journal of Occupational Therapy. 2000;63(6):277-282.
8. Ayres AJ. Sensory Integration and the child. California, USA: Western Psychological Services; 1979.

9. Dunn W. Sensory Profile. Psychological corporation, Texas, USA: 1999.

10. Bruininks RH. Bruinink- Oseretsky Test of Motor Proficiency. Circle Pines, USA: American Guidance Service; 1978.

11. Sparrow SS, Balla DA, Cicchetti DV. Vineland Adaptive Behavior Scales: Survey form manual. Circle Pines, USA; American Guidance Service; 1984.

12. Satiansukpong N, Pongsaksri M, Sung US, et al. Thai elephant-assisted therapy program: the feasibility in assisting an individual with autism. World Federation of Occupational Therapy Bulletin. 2008;58(1):17-26.

13. Satiansukpong N, Pongsaksri M, Sasat D. Thai elephant assisted therapy program in children with down syndrome. Occup Ther Int. 2016;23(2):121131.

14. Beery KE, Beery NA. The Beery-Buktenica Developmental Test of VisualMotor Integration. Pearson clinical assessment, USA: San Antonio; 2010. 